\title{
Prevalência de sobrepeso e obesidade em escolares da rede pública do município de Jundiaí, São Paulo
}

\author{
Prevalence of overweight and obesity among children of public schools in the city of Jundiaí, São Paulo, Brazil
}

\author{
Amanda Somolanji Vanzelli', Camille Tavares de Castro ${ }^{1}$, Melina da Silva Pinto ${ }^{1}$, Saulo Duarte Passos ${ }^{2}$
}

\section{RESUMO}

Objetivo: Determinar a prevalência de obesidade de acordo com o nível socioeconômico e o sexo, em escolares do ensino fundamental, segundo dois critérios diagnósticos.

Métodos: Realizou-se um estudo transversal nas escolas públicas de ensino fundamental de Jundiaí, São Paulo, com 662 alunos de quinta a oitava séries, com idades entre dez e 18 anos, em 2005. A amostra foi selecionada por meio de procedimento sistemático, de acordo com a série e o sexo. Calculou-se o índice de massa corpórea (IMC). Os pontos de corte para classificação do estado nutricional foram escolhidos de acordo com dois critérios antropométricos: valores de IMC em percentil e valor numérico de IMC como ponto de corte.

Resultados: A prevalência de sobrepeso foi $17 \%$ quando se utilizou o critério de percentil e $13 \%$ ao usar o corte por valor numérico. A prevalência de obesidade foi 8 e $9 \%$, respectivamente. Houve diferença quanto ao sexo, quando se utilizou a classificação por percentil, com maior percentual de magreza encontrado no sexo masculino. O sobrepeso e a obesidade foram mais observados em adolescentes de melhor condição socioeconômica.

Conclusões: A prevalência de sobrepeso e obesidade foi eleva$\mathrm{da}$, sendo maior nas classes mais favorecidas. A medida do IMC por percentil detectou maior número de adolescentes com sobrepeso e obesidade, comparada à classificação por valor numérico.

Palavras-chave: obesidade; sobrepeso; epidemiologia; saúde do adolescente.

\section{ABSTRACT}

Objective: To determine the prevalence of obesity related to gender and socioeconomic level in students of public schools, according to two different nutritional criteria.

Methods: A cross-sectional study was performed in public schools of the city of Jundiaí, São Paulo, Brazil, and included 662 students with 10-18 years old that attended $5^{\text {th }}-8^{\text {th }}$ grades, in 2005 . The sample was selected using systematic procedure, according to the student's grade and gender. The body mass index (BMI) was calculated and cutoff points were selected for classification of nutritional state according to two anthropometrics criteria: values of BMI as percentiles and as sharp cutoff point.

Results: The prevalence of overweight was $17 \%$ using the percentile criteria and $13 \%$ with the numerical cutoff. The frequency of obesity was 8 and $9 \%$, respectively for both criteria. Difference between genders was found using the percentile criteria, with higher percentage of leanness in males. Overweight and obesity were more frequent among adolescents of better socioeconomic status.

Conclusions: The prevalence of overweight and obesity were higher in students of better economic classes. The BMI percentiles detected a higher number of adolescents with overweight and obesity compared to the numerical cutoff.

Key-words: obesity; overweight; epidemiology; adolescent health.

\footnotetext{
${ }^{1}$ Acadêmica da Faculdade de Medicina de Jundiaí (FMJ), Jundiaí, SP, Brasil ${ }^{2}$ Professor-associado do Departamento de Pediatria da FMJ e livre-docente na área Materno Infantil da Faculdade de Saúde Pública da Universidade de São Paulo, Jundiaí, SP, Brasil
}

Endereço para correspondência: Amanda Somolanji Vanzelli

Rua Arnaldo Simões Pinto, 166
CEP 02917-110 - Pirituba/SP
E-mail: amandafmj34@yahoo.com.br

Fonte financiadora do projeto: Conselho Nacional de Desenvolvimento Científico e Tecnológico (CNPq)

Recebido: 3/6/2007

Aprovado: 15/1/2008 


\section{Introdução}

A obesidade vem aumentando de forma alarmante, sendo considerada uma verdadeira epidemia mundial que atinge todas as faixas etárias, em especial, as crianças ${ }^{(1)}$. Trata-se de uma doença crônica, definida como excesso de gordura corporal $^{(2,3)}$, na qual ocorre concomitância de fatores de risco genéticos e ambientais ${ }^{(4,5)}$. Há possibilidade de associação com outras patologias, como a síndrome plurimetabólica, com risco elevado de desenvolvimento de hipertensão, dislipidemia e diabetes melito tipo $2^{(6)}$. O aumento do consumo de alimentos gordurosos, com alta densidade energética, e a diminuição na prática de exercícios físicos são os dois principais fatores ligados ao meio ambiente que colaboram para o aumento da prevalência da obesidade ${ }^{(6)}$. Além disso, fatores como o equilíbrio entre consumo e acúmulo de gordura corporal, que podem ser geneticamente regulados, contribuem para a obesidade ${ }^{(7)}$.

A antropometria permite monitorar o estado nutricional com boa acurácia, desde que as técnicas de medidas sejam adequadas, com o benefício de se tratar de método de baixo custo, não invasivo, universalmente aplicável e com boa aceitação pela população ${ }^{(8)}$. As relações que levam em consideração peso e altura apresentam grande precisão, porque tais medições oferecem baixa margem de erro. O índice de massa corpórea (IMC) é o mais utilizado; há necessidade, porém, de determinar os pontos de corte para avaliar sobrepeso e obesidade específicos para cada população, de acordo com suas características de desenvolvimento, por meio de estudos longitudinais ${ }^{(8)}$. No Brasil, não há estudos longitudinais com adolescentes; assim, são utilizados os pontos de corte estabelecidos para a população americana pelo National Center for Health Statistics (NCHS) e recomendados como referência pela Organização Mundial de Saúde (OMS) ${ }^{(9)}$.

Um dos índices mais utilizados para a avaliação do sobrepeso em crianças e adolescentes na rotina pediátrica e em Saúde Pública $^{(10)}$ é a distribuição percentilar proposta por Must et al ${ }^{(11)}$ para o IMC, que foi elaborada para classificar crianças a partir de seis anos e adultos de acordo com sexo, idade e raça. Segundo Must et al $l^{(11)}$, define-se como sobrepeso crianças com IMC entre os percentis 85 e 95 e, como obesas, aquelas com IMC acima do percentil 95. Outro critério utilizado para avaliar o estado nutricional é a curva de Cole et al $l^{(12)}$, baseada em um grupo de estudos do perfil do IMC por idade em vários países, inclusive o Brasil, que estabeleceu limites para sobrepeso e obesidade na faixa etária de dois a 20 anos para uso internacional.

Apesar de bastante utilizado, tanto para a população americana quanto internacionalmente, e de ser recomendado pela OMS, o critério de Must et al ${ }^{(11)}$ baseia-se exclusivamente no padrão de referência da população americana e deve ser considerado com cautela, em virtude da elevada prevalência de obesidade naquela população. Já o critério de Cole et $a^{(12)}$, com definições de sobrepeso e obesidade em crianças e adolescentes com base no IMC, disponibiliza pontos de corte específicos por sexo e idade correspondentes, respectivamente, aos IMC 25 e $30 \mathrm{~kg} / \mathrm{m}^{2}$ na idade de 18 anos. Tais autores incluíram dados de crianças e adolescentes de seis países (Estados Unidos, Brasil, Grã-Bretanha, Hong Kong, Holanda e Cingapura), constituindo-se, talvez, em critério mais adequado para comparações internacionais ${ }^{(13)}$.

A ausência de unanimidade nos critérios apontados na literatura para definir obesidade infantil indica a necessidade de estabelecer pontos de corte específicos para cada população. Assim, o presente estudo pretende comparar a prevalência de obesidade em um grupo de escolares da rede pública estadual do município de Jundiaí, segundo os dois critérios diagnósticos mais utilizados nas práticas assistenciais e em pesquisa, no sentido de avaliar as diferentes estimativas de prevalência de obesidade em nível clínico e populacional e as relações com as condições socioeconômicas.

\section{Métodos}

Estudo transversal para avaliar a prevalência de sobrepeso, obesidade e desnutrição em adolescentes de dez a 18 anos, matriculados na quinta a oitava séries do ensino fundamental de todas as 33 escolas públicas estaduais do município de Jundiaí, no ano de 2005. Os dados foram coletados em dezembro de 2005.

Para cálculo do tamanho da amostra, utilizou-se um banco de dados fornecido pela Secretaria Estadual de Educação, com 18.077 adolescentes matriculados de quinta a oitava séries em escolas públicas do município de Jundiaí. Como interessava ao estudo apenas crianças com idade entre dez e 18 anos, foram excluídas as demais. O tamanho inicial da amostra para o estudo de prevalência calculado foi de 606 alunos, aceitando que a proporção de obesos seria de $9 \%$, com margem de erro de $3 \%$, e considerando a possibilidade de perda de até $20 \%$ em cada estrato. O processo de amostragem se deu por meio de técnica de multiestágio: primeiro se realizou a amostragem estratificada proporcional ao número de alunos de cada escola, seguida de amostragem aleatória dentro de cada escola, incluindo-se no estudo todas as escolas urbanas públicas do município. Esse processo amostral permitiu que cada escolar tivesse probabilidade igual de ser sorteada, com reposição das perdas de até $20 \%$. 
Dos adolescentes elegíveis, foi determinado o estado nutricional de 662, cujos pais autorizaram a participação e assinaram o termo de consentimento, após receberem uma carta explicativa sobre o estudo. A pesquisa foi aprovada pela Comissão de Ética em Pesquisa da Faculdade de Medicina de Jundiaí. A coleta de dados foi feita por meio de preenchimento de um protocolo padrão, aplicado por acadêmicos bolsistas de Medicina, após treinamento para padronizar o processo de mensuração. Os entrevistadores foram orientados a registrar o peso conforme a última unidade completa em quilogramas.

Para pesar as crianças, foram utilizadas balanças eletrônicas da marca Glicomed $^{\circledR}$, com capacidade de $150 \mathrm{~kg}$ e precisão de $50 \mathrm{~g}$. Os alunos foram examinados nas escolas em que estudavam e pesados vestindo apenas roupas leves e descalços, permanecendo eretos, no centro da balança, com os braços esticados ao lado do corpo, sem se movimentar ${ }^{(9,14)}$.

Para a medida da estatura, as crianças foram colocadas descalças, em posição ereta, encostadas numa superfície plana vertical, braços pendentes com as mãos espalmadas sobre as coxas, os calcanhares unidos e as pontas dos pés afastadas, formando ângulo de $60^{\circ}$, joelhos em contato, cabeça ajustada ao plano de Frankfurt e calcanhares, ombros e nádegas encostados na parede ${ }^{(9,14)}$, em inspiração profunda. A medida foi feita em triplicata, para o cálculo do valor médio, utilizando-se um estadiômetro de alumínio tipo trena fixado à parede, com capacidade de $2 \mathrm{~m}$ e precisão de $0,1 \mathrm{~cm}$.

A partir do cálculo do IMC, ou seja, dividindo-se o peso $(\mathrm{kg})$ pela altura $(\mathrm{m})$ ao quadrado $\left(\mathrm{IMC}=\mathrm{kg} / \mathrm{m}^{2}\right)$, foram utilizados os pontos de corte propostos por Must et al ${ }^{(11)}$, que definem como sobrepeso crianças com IMC entre os percentis 85 e 95 e, como obesas, acima do percentil 95. Foram empregados também pontos de corte propostos por Cole et al ${ }^{(12)}$, estimados de forma que os pontos das curvas ajustadas dos percentis $85 \mathrm{e}$ 95 de IMC aos18 anos fossem obrigatoriamente os pontos de corte para sobrepeso e obesidade utilizados para adultos (25 e 30, respectivamente).

A classificação econômica das famílias foi avaliada de acordo com o Critério de Classificação Econômica do Brasil (CCEB) (classes A1, A2, B1, B2, C, D e E), que leva em consideração a posse de bens de consumo, grau de instrução do chefe da família e a presença de empregada mensalista, sendo a categoria "A" considerada como melhor nível econômico(15).

Os dados coletados foram digitados e armazenados em um banco de dados desenvolvido no software Epi-Info. As prevalências de sobrepeso e obesidade foram calculadas por proporção e as diferenças entre os sexos e entre as faixas etárias foram analisadas por meio do teste do qui-quadrado ou qui-quadrado de Yates, considerando-se significante $p \leq 0,05$. O software utilizado para análise dos dados foi o SAS versão 8.2.

\section{Resultados}

Foram examinados 662 escolares, sendo 326 (49\%) do sexo masculino e $336(51 \%)$ do feminino, com predomínio na faixa de 11 a 15 anos (97\%). Segundo a classificação socioeconômica adotada, 16 (3\%) famílias encontravam-se na classe A, $74(11 \%)$ na classe B1, 150 (23\%) na classe B2, 309 (47\%) na classe C, $110(17 \%)$ na classe D e três (1\%) na classe E - ou seja, apenas $90(14 \%)$ nas classes mais favorecidas.

Com base no critério proposto por Cole et al ${ }^{(12)}$, a prevalência de obesidade na população estudada foi $8 \%$ e a de sobrepeso foi $16 \%$, enquanto que, pelo critério de Must

Tabela 1 - Prevalência de obesidade por sexo, segundo Cole et a/(11)

\begin{tabular}{lcccccc}
\hline & \multicolumn{2}{c}{ Eutrofia } & \multicolumn{2}{c}{ Sobrepeso } & \multicolumn{2}{c}{ Obesidade } \\
& $\mathbf{n}$ & $\mathbf{\%}$ & $\mathbf{n}$ & $\mathbf{0}$ & $\mathbf{n}$ & $\mathbf{\%}$ \\
\hline Masculino & 257 & 79 & 44 & 14 & 25 & 8 \\
Feminino & 246 & 73 & 64 & 19 & 26 & 8 \\
Total & $\mathbf{5 0 3}$ & $\mathbf{7 6}$ & $\mathbf{1 0 8}$ & $\mathbf{1 6}$ & $\mathbf{5 1}$ & $\mathbf{8}$ \\
\hline
\end{tabular}

Qui-quadrado: $p=0,1485$

Tabela 2 - Prevalência de obesidade por sexo, segundo Must et a/(10)

\begin{tabular}{lcccccccccc} 
& \multicolumn{2}{c}{ Desnutrição } & \multicolumn{2}{c}{$\begin{array}{c}c \\
\text { nigilância }\end{array}$} & \multicolumn{2}{c}{ Eutrofia } & \multicolumn{2}{c}{ Sobrepeso } & \multicolumn{2}{c}{ Obeso } \\
& $\mathbf{n}$ & $\mathbf{\%}$ & $\mathbf{n}$ & $\mathbf{\%}$ & $\mathbf{n}$ & $\mathbf{\%}$ & $\mathbf{n}$ & $\mathbf{\%}$ & $\mathbf{n}$ & $\mathbf{\%}$ \\
\hline Masculino & 19 & 6 & 28 & 9 & 209 & 64 & 40 & 12 & 30 & 9 \\
Feminino & 11 & 3 & 9 & 3 & 241 & 72 & 46 & 14 & 29 & 9 \\
Total & $\mathbf{3 0}$ & $\mathbf{5}$ & $\mathbf{3 7}$ & $\mathbf{6}$ & $\mathbf{4 5 0}$ & $\mathbf{8 3}$ & $\mathbf{8 6}$ & $\mathbf{1 3}$ & $\mathbf{5 9}$ & $\mathbf{9}$ \\
\hline
\end{tabular}

Qui-quadrado: $p=0,006$ 
et al ${ }^{(11)}$, houve $9 \%$ de obesidade, $13 \%$ de sobrepeso e $5 \%$ de desnutrição. As Tabelas 1 e 2 mostram existir diferença significante entre crianças eutróficas e com sobrepeso e entre as últimas e as obesas pelo critério de Must et al ${ }^{(11)}$, não se verificando a mesma diferença significativa quando a classificação nutricional é feita pelo critério de Cole et al ${ }^{(12)}$. Para as categorias determinadas pelo critério de Must et al ${ }^{(11)}$, houve diferença entre os sexos, com percentual de magreza mais elevado em homens e de sobrepeso em mulheres.
De acordo com a classificação socioeconômica, observouse um predomínio de escolares com sobrepeso e obesidade nas classes A e B, comparativamente ao número de escolares eutróficos (Tabelas 3 e 4). A Tabela 5 mostra a concordância entre os dois critérios antropométricos propostos para avaliar a obesidade na adolescência. Com o critério proposto por Must et al ${ }^{(11)}$, obteve-se maior número de casos de obesidade, enquanto que o proposto por Cole et al ${ }^{(12)}$ classificou maior número na categoria de sobrepeso.

Tabela 3 - Prevalência de sobrepeso e obesidade de acordo a classificação socioeconômica, segundo Cole et a/(11)

\begin{tabular}{lcccccc}
\hline & \multicolumn{2}{c}{ Eutrofia } & \multicolumn{2}{c}{ Sobrepeso } & \multicolumn{2}{c}{ Obesidade } \\
& $\mathbf{n}$ & $\%$ & $\mathbf{n}$ & $\%$ & $\mathbf{n}$ & $\%$ \\
\hline A1 $(n=1)$ & 1 & 100 & 0 & 0 & 0 & 0 \\
A2 $(n=15)$ & 10 & 67 & 2 & 13 & 3 & 20 \\
B1 $(n=74)$ & 13 & 18 & 42 & 57 & 19 & 26 \\
B2 $(n=150)$ & 91 & 61 & 40 & 27 & 19 & 13 \\
C $\quad(n=309)$ & 280 & 91 & 19 & 6 & 10 & 3 \\
D $\quad(n=110)$ & 105 & 95 & 5 & 5 & 0 & 0 \\
E $\quad(n=3)$ & 3 & 100 & 0 & 0 & 0 & 0 \\
\hline
\end{tabular}

Qui-quadrado de Yates: $p<0,0001$

Tabela 4 - Prevalência de sobrepeso e obesidade de acordo com a classificação socioeconômica, segundo Must et al(10)

\begin{tabular}{|c|c|c|c|c|c|c|c|c|c|c|}
\hline & \multicolumn{2}{|c|}{ Desnutrição } & \multicolumn{2}{|c|}{$\begin{array}{l}\text { Vigilância } \\
\text { nutricional }\end{array}$} & \multicolumn{2}{|c|}{ Eutrofia } & \multicolumn{2}{|c|}{ Sobrepeso } & \multicolumn{2}{|c|}{ Obesidade } \\
\hline & n & $\%$ & $\mathbf{n}$ & $\%$ & $\mathbf{n}$ & $\%$ & $\mathbf{n}$ & $\%$ & $\mathbf{n}$ & $\%$ \\
\hline A1 $(n=1)$ & 0 & 0 & 0 & 0 & 1 & 100 & 0 & 0 & 0 & 0 \\
\hline A2 $(n=15)$ & 0 & 0 & 0 & 0 & 2 & 13 & 10 & 67 & 3 & 20 \\
\hline B1 $(n=74)$ & 0 & 0 & 0 & 0 & 18 & 24 & 28 & 38 & 28 & 38 \\
\hline B2 $(n=150)$ & 0 & 0 & 4 & 3 & 93 & 61 & 32 & 21 & 21 & 14 \\
\hline C $\quad(n=309)$ & 12 & 4 & 23 & 8 & 256 & 83 & 11 & 4 & 7 & 2 \\
\hline$D \quad(n=110)$ & 15 & 14 & 10 & 9 & 80 & 73 & 5 & 5 & 0 & 0 \\
\hline$E \quad(n=3)$ & 3 & 100 & 0 & 0 & 0 & 0 & 0 & 0 & 0 & 0 \\
\hline
\end{tabular}

Qui-quadrado de Yates: $p<0,0001$

Tabela 5 - Correlação da classificação antropométrica entre os critérios de Cole et a/(11) e Must et a/ ${ }^{(10)}$, expressa por número de crianças em cada categoria de classificação

\begin{tabular}{|c|c|c|c|c|c|}
\hline Cole & Must & Eutrofia & Sobrepeso & Obesidade & Total \\
\hline Desnutrido & & 30 & 0 & 0 & 30 \\
\hline Vigilância & & 37 & 0 & 0 & 37 \\
\hline Eutrofia & & 434 & 16 & 0 & 450 \\
\hline Sobrepeso & & 2 & 84 & 0 & 86 \\
\hline Obesidade & & 0 & 8 & 51 & 59 \\
\hline Total & & 503 & 108 & 51 & 662 \\
\hline
\end{tabular}

Coeficiente de concordância kappa=0,894 (IC95\%=0,854-0,933) 


\section{Discussão}

A prevalência de sobrepeso e obesidade foi elevada, com valores similares às pesquisas anteriores realizadas no Brasil ${ }^{(16,17)}$ e em países desenvolvidos ${ }^{(18-20)}$. A importância deste achado foi demonstrada em um estudo que evidenciou que $40 \%$ das crianças obesas até os sete anos tornam-se adultos obesos e que 70 a $80 \%$ de adolescentes obesos tornam-se adultos obesos, além de possuírem maiores riscos de morbimortalidade ${ }^{(2)}$.

No Brasil, segundo dados da Associação Nacional de Empresas de Pesquisa, a maior parte das famílias pertence às classes socioeconômicas de menor renda (classes D, 33\% e E, 31\%); porém, os adolescentes mais atingidos pela obesidade ainda pertencem às classes sociais mais privilegiadas. Apesar da tendência recente de mudança nesse perfil ${ }^{(16)}$, com o aumento progressivo das taxas de obesidade nas classes menos favorecidas, o presente estudo corrobora resultados de pesquisas anteriores ${ }^{(17,18)}$, com maior prevalência de obesidade nas classes mais favorecidas.

Ao utilizar os critérios de Cole et al ${ }^{(12)}$, não foram observadas diferenças significativas na prevalência de sobrepeso e obesidade entre os sexos. Entretanto, ao adotar os critérios de Must et al ${ }^{(11)}$, notou-se percentual de magreza mais elevado nos homens e de sobrepeso nas mulheres, concordante com a literatura ${ }^{(21)}$. Em estudo de caso em adolescentes do sexo masculino, observou-se que o risco de mortalidade por doenças cardiovasculares foi maior no grupo com IMC $>25^{(22)}$.

Vários estudos brasileiros têm mostrado maior prevalência de excesso de peso em adolescentes do sexo feminino. Na Pesquisa Nacional de Saúde e Nutrição, a prevalência de sobrepeso foi aproximadamente duas vezes maior em adolescentes do sexo feminino $(10,5 \%)$ em relação ao masculino $(4,8 \%)^{(23,24)}$. Os resultados do presente trabalho concordam com estes achados ${ }^{(21)}$ e ratificam outros estudos ${ }^{(17)}$. Entretanto, vale ressaltar que existem resultados discordantes, com maior prevalência de sobrepeso e obesidade no sexo masculino em relação ao feminino ${ }^{(18,25)}$.

Existe consenso quanto ao padrão diferenciado do estado antropométrico entre os sexos. Sabe-se que, na puberdade, ocorre maior crescimento de tecido muscular nos homens pela ação da testoterona e, nas mulheres, de tecido adiposo, por ação estrogênica ${ }^{(18,21)}$; entretanto, os fatores comportamentais, principalmente aqueles relacionados à alimentação e ao estilo de vida, têm contribuído para a ocorrência de sobrepeso e obesidade em ambos os sexos ${ }^{(24,26)}$.

Flegal et $\mathrm{al}^{(27)}$, em estudo comparativo sobre a prevalência de sobrepeso e obesidade por meio da curva de crescimento de IMC do Centers for Diseases Control and Prevention com os valores propostos por Cole et $a l^{(12)} \mathrm{e}$ Must et $a l^{(11)}$, encontraram prevalências diferentes de acordo com os critérios utilizados. Tais diferenças podem ser explicadas pelos diversos métodos de estimativa e suavização das curvas percentilares e, principalmente, pela população de referência utilizada para o conjunto de dados da amostra. Desta forma, as prevalências encontradas pelo critério de Must $e t$ a ${ }^{(11)}$ são mais elevadas do que as encontradas pelo outro critério. Da mesma forma, Oliveira et a ${ }^{(13)}$ observaram, em estudo sobre a acurácia do diagnóstico de obesidade em adolescentes, que o procedimento proposto por Must fornece diagnóstico precoce de risco antropométrico para obesidade.

A interpretação de medidas antropométricas em adolescentes que visam avaliar a obesidade deve considerar que a mudança da categoria de obeso para não obeso pode ocorrer por modificações da composição corporal próprias do processo de crescimento, nem sempre incorporadas nos pontos de corte das distribuições de referência, uma vez que há certa variabilidade entre indivíduos. Tal afirmativa sedimenta-se no fato de que todos os procedimentos antropométricos existentes tentam estimar a proporção de massa gorda indicativa de obesidade e que, por serem de natureza transversal e não avaliarem velocidade de crescimento e variações dos IMC, são limitados no que tange à variabilidade da aceleração do crescimento nos diferentes indivíduos ${ }^{(27)}$.

Conclui-se que a prevalência de sobrepeso e obesidade no presente estudo foi alta, devendo ser esta problemática entendida como emergente e preocupante. Há necessidade de estudo em nível nacional nesse grupo etário para verificar a dimensão do problema, fornecendo, assim, subsídios para as estratégias de prevenção e controle, com implantação de ações individuais e coletivas, onde quer que esses adolescentes estejam inseridos.

\section{Agradecimentos}

À professora Regina Beatriz M. Mostério, assessora pedagógica da FMJ, pela dedicação, apoio e incentivo. 


\section{Referências bibliográficas}

1. Autoria não referida. Obesity: preventing and managing the global epidemic. Report of a WHO consultation. World Health Organ Tech Rep Ser 2000;894:i-xii,1-253.

2. Guillaume M. Defining obesity in childhood: current practice. Am J Clin Nutr 1999;70:126S-30.

3. Zlochevsky ER. Obesidade na infância e adolescência. Rev Paul Pediatr 1996;14:124-33.

4. Blair SN, Horton E, Leon AS, Lee IM, Drinkwater BL, Dishman RK et al. Physical activity, nutrition, and chronic disease. Med Sci Sports Exerc 1996;28:335-

5. Egger G, Bolton A, O'Neill M, Freeman D. Effectiveness of an abdominal obesity reduction programme in men: the GutBuster "waist loss' programme. Int J Obes Relat Metab Disord 1996;20:227-31.

6. Dietz WH. Health consequences of obesity in youth: childhood predictors of adult disease. Pediatrics 1998;101:518S-25.

7. Rosenbaum M, Leibel RL. The physiology of body weight regulation: relevance to the etiology of obesity in children. Pediatrics 1997;101:525-39.

8. Dietz WH, Bellizzi MC. Introduction: the use of mass index to assess obesity in children. Am J Clin Nutr 1999;70:123S-5S.

9. Autoria não referida. Physical Status: the use and interpretation of anthropometry. Report of a WHO Expert Committee. World Health Organ Tech Rep Ser 1995;854:1-452.

10. Balaban G, Silva GA. Overweight and obesity prevalence in children and adolescents from a private school in Recife. J Pediatr (Rio J) 2001;77:96-100.

11. Must A, Dallal GE, Dietz WH. Reference data for obesity: 85th and 95th percentiles of body mass index (wt/ht2) and triceps skinfold thickness. Am J Clin Nutr 1991;53:839-46

12. Cole TJ, Bellizzi MC, Flegal KM, Dietz WH. Establishing a standard definition for child overweight and obesity worldwide: international survey. BMJ 2000;320:1240-3.

13. Oliveira FL, Taddei JA, Escrivão MA, Cobayashi F, Barros ME, Vítolo MR et al. Accuracy of obesity diagnosis in Brazilian adolescents: comparison of Cole et al and Must et al criteria with DXA percentage of fat mass. Nutr Hosp 2006;21:484-90.

14. Jelliffe DB, editor. Evaluación del estado de nutrición de la comunidad. Série de monografías, 53. Genebra: Organização Mundial de Saúde; 1968.
15. Associação Nacional de Empresas de Pesquisa [homepage on the Internet] Classificação Econômica Brasil do ANEP - 2002 [cited 2002 May 5]. Available from: http://www.abep.org/codigosguias/Criterio_Brasil_2008.pdf

16. Monteiro CA, Conde WL. A tendência secular da obesidade segundo estratos sociais: Nordeste e Sudeste do Brasil, 1975-1989-1997. Arq Bras Endocrinol Metabol 1999;43:186-94.

17. Vieira VC, Priori SE, Ribeiro SM, Franceschini SC, Almeida LP. [Socioeconomic, nutritional and health profile of adolescents recently admitted to a Brazilian public university]. Rev Nutr 2002;15:273-82.

18. Costa COM. Crescimento e desenvolvimento na infância e adolescência. In: Costa COM, Souza RP, editores. Avaliação e cuidados primários da criança e do adolescente. Porto Alegre: Artes Médicas; 1998. p. 35-45.

19. Anta RM, Carvajales PA, Marcos AM, SobalerAM, Sobrado RR, Gonzáles-Fernández M. Hábitos alimentarios y ingesta de energia y nutrientes en adolescentes con sobrepeso en comparación con los de peso normal. An Esp Pediatr 1996;44:203-8.

20. Brugman E, Meulmeester JF, Spee-van der Weeker A, Beuker RJ, Zaadstra $\mathrm{BM}$, Radder JJ et al. Dieting, weight and health in adolescents in The Netherlands. Int J Obes Relat Metab Disord 1997;21:54-60.

21. Marshall WA, Tanner JM. Puberty. In: Falkner F, Tanner JM, editors. Human growth: a comprehensive treatise. Volume 2: postnatal growth; neurobiology. $2^{\text {nd }}$ ed. New York: Plenum Press; 1986. p. 171-210.

22. Nuzzo L. Avaliação do estado nutricional de adolescentes de uma instituição particular de ensino [tese de mestrado]. São Paulo (SP): Unifesp; 1998.

23. Brasil-Ministério da Saúde. Condições nutricionais da população brasileira: adultos e idosos. Pesquisa Nacional de Saúde e Nutrição. Brasília: Ministério da Saúde; 1991.

24. Neutzling MB. Sobrepeso em adolescentes brasileiros - Brasil, PNSN-1989 [tese de mestrado]. São Paulo (SP): Unifesp; 1998.

25. Fonseca VM, Sichieri R, Veiga GV. Factors associated with obesity among adolescents. Rev Saude Publica 1998;32:541-9.

26. Eisenstein E. Atraso puberal e desnutrição crônica primária [tese de mestrado] São Paulo (SP): Unifesp; 1999.

27. Flegal KM, Ogden CL, Wei R, Kuczmarski RL, Johnson CL. Prevalence of overweight in US children: comparison of US growth charts from the Centers for Diseases Control and Prevention with other reference values for body mass index. Am J Clin Nutr 2001;73:1086-93. 